\title{
Netrin-4 Promotes Differentiation and Migration of Osteoblasts
}

\author{
YUICHIRO ENOKI ${ }^{1}$, TSUYOSHI SATO ${ }^{1}$, SHOICHIRO KOKABU ${ }^{1,2}$, NAOKI HAYASHI $^{1}$, \\ TAKANORI IWATA $^{3}$, MASAYUKI YAMATO ${ }^{3}$, MICHIHIKO USUI $^{4}$, MASAHITO MATSUMOTO $^{5}$, \\ TAKETO TOMODA $^{1}$, WATARU ARIYOSHI ${ }^{6}$, TATSUJI NISHIHARA ${ }^{6}$ and TETSUYA YODA ${ }^{1}$ \\ ${ }^{1}$ Department of Oral and Maxillofacial Surgery, Saitama Medical University, Saitama, Japan; \\ ${ }^{2}$ Division of Molecular Signaling and Biochemistry, \\ Department of Health Promotion and Kyushu Dental University, Fukuoka, Japan; \\ ${ }^{3}$ Institute of Advanced Biomedical Engineering and Science, Tokyo Women's Medical University, Tokyo, Japan; \\ ${ }^{4}$ Division of Periodontology, Department of Cardiology and Periodontology, \\ Kyushu Dental University, Fukuoka, Japan; \\ ${ }^{5}$ Division of Functional Genomics and Systems Medicine, \\ Research Center for Genomic Medicine, Saitama Medical University, Saitama, Japan; \\ ${ }^{6}$ Division of Infections and Molecular Biology, \\ Department of Health Promotion, Kyushu Dental University, Fukuoka, Japan
}

\begin{abstract}
Background/Aim: While netrin-4 plays a vital role in the vascular system, the role of netrin-1 in osteoblast differentiation is not well understood. In this study we explored whether netrin-4 has functional roles in osteoblasts. Materials and Methods: Quantitative reverse-transcriptase polymerase chain reaction (PCR), RNA interference, the generation of plasmids, transfections, measurement of alkaline phosphatase activity, a mineralization assay, a migration assay and a cell proliferation assay were performed. Results: Netrin-4 expression was up-regulated during osteoblast differentiation and an RNA interference experiment showed that small interfering RNA used to silence netrin-4 inhibited osteoblast differentiation. Recombinant mouse netrin-4 promoted alkaline phosphatase $(A L P)$ activity of osteoblasts and enhancement of calcium deposits. Moreover, we constructed a vector containing the netrin-4 gene on the basis of the plasmid pcDNA3.1/V5-His. Overexpression of netrin-4 enhanced differentiation of osteoblasts. Finally, recombinant mouse netrin-4 promoted cell migration of osteoblasts. Conclusion: Netrin-4 promotes differentiation and migration of osteoblasts.
\end{abstract}

This article is freely accessible online.

Correspondence to: Prof. Tsuyoshi Sato, Department of Oral and Maxillofacial Surgery, Saitama Medical University, 38 Morohongou, Moroyama-machi, Iruma-gun, Saitama, 350-0495, Japan. Tel/Fax: +81492761859, e-mail: tsato@saitama-med.ac.jp

Key Words: Netrin-4, osteoblast, differentiation, migration.
Bone homeostasis refers to the balance between the formation by osteoblasts and the degradation by osteoclasts (1). The function of these cells is regulated by several factors which other organs produce to communicate with bones. Indeed, the vessels and nerves inside bones are essential for bone development and remodeling (2-4). Previous studies have demonstrated that neural and vascular regulation factors, such as semaphorins and netrins, orchestrate bone metabolism. For instance, semaphorin 3A not only promotes osteoblast differentiation, but also inhibits osteoclast differentiation and semaphorin 7A is capable of increasing the migration of osteoblasts $(5,6)$. Semaphorin $4 \mathrm{D}$, derived from osteoclasts, potently inhibits osteoblast differentiation (7). Moreover, a recent study showed that netrin-1 plays a crucial role in osteoclast differentiation $(8,9)$.

Mammalian netrins including the secreted proteins (netrin-1, netrin-3, netrin-4 and netrin-5) and membrane-bound proteins (netrin-G1 and netrin-G2) are involved in the regulation of both axon guidance and angiogenesis (10). Among these netrins, netrin-4 (Ntn4) has several functions, such as promoting neurite extension, regulating pulmonary airway branching, vasculogenesis patterning, endothelial proliferation in pathological angiogenesis, and negative regulation of vascular branching in the retina (11-13). A recent study using human samples demonstrated that Ntn4 expression is an independent predictor of improved outcome in breast cancer (14). We have previously demonstrated that $\mathrm{Ntn} 4$, that is derived from vascular endothelial cells, inhibits osteoclast differentiation (15). As the remodeling of bone tissue is regulated by osteoclasts and osteoblasts, the question whether Ntn4 affects cellular function in osteoblasts has been raised. However, little is known about 
the role of Ntn4 in osteoblasts. In this study, we explored whether Ntn4 affects the function of osteoblasts.

\section{Materials and Methods}

Reagents. Recombinant mouse Ntn4 (1132-N4) was purchased from R\&D systems (Minneapolis, MN, USA).

Cell culture. The mouse pre-osteoblastic cell line MC3T3-E1 which was kindly provided from Dr. Toru Ogasawara (Department of Oral and Maxillofacial Surgery, The University of Tokyo, Japan) was maintained in $\alpha$-modified minimum essential medium ( $\alpha$-MEM) (WAKO, Osaka, Japan) with $10 \%$ fetal bovine serum (FBS) (BioWest, Nuaillé, France). For osteoblastic differentiation assay, cells were cultured in the medium containing ascorbic acid (50 $\mu \mathrm{g} / \mathrm{mL})$ and $\beta$-glycerophosphate $(10 \mathrm{mM})$. The medium was changed every 3 days. All cultures were maintained at $37^{\circ} \mathrm{C}$ in humidified air including $5 \% \mathrm{CO}_{2}$.

Cell proliferation assay. The cells were incubated with recombinant $\mathrm{Ntn} 4$ at the indicated concentration for 3 days. The sample cells were quantified using a Cell Titer 96 Aqueous One Solution Cell Proliferation Assay (Promega, Madison, WI, USA), according to the manufacturer's instructions. The measurements are represented by the means of at least three independent experiments, with each data point based on six replicates.

Measurement of alkaline phosphatase (ALP) activity. Cells were plated at a density of $2 \times 10^{4}$ cells in 24 -well plates. After reaching confluence, cells were incubated with ascorbic acid $(50 \mathrm{~g} / \mathrm{mL})$ and $\beta$-glycerophosphate $(10 \mathrm{mM})$ in the presence or absence of recombinant Ntn4 at the indicated concentration for 7 days. ALP activity was assayed (WAKO, Tokyo, Japan) as previously described. The measurements are expressed as the means of three independent experiments, with each data point based on four replicates.

Mineralization assay. Assay was performed as previously described (16). Cells were inoculated at $10 \times 10^{4}$ cells in 6-well plates. After reaching confluence, the cells were incubated with $\beta$ glycerophosphate $(10 \mathrm{mM})$ in the presence or absence of recombinant Ntn4 at the indicated concentration for 21 days. Mineralization of the cells was determined by alizarin red staining. The cells were fixed with ice-cold $70 \%$ ethanol and stained with alizarin red to detect calcification.

Migration assay. Migration of cells was quantified using the Oris ${ }^{\mathrm{TM}}$ Cell Migration Assay kit (Platypus Technologies, LLC, Madison, WI) according to the manufacturer's protocol. Briefly, a 96-well plate was populated with silicone stoppers and cells were added to each well. When the cells were confluent, the stoppers were removed and the clear area was defined as the area before migration. The cells were incubated with recombinant $\mathrm{Ntn} 4$ at the indicated concentration for $12 \mathrm{~h}$. The cells were fixed in $4 \%$ paraformaldehyde for $10 \mathrm{~min}$ at room temperature, rinsed in phosphate-buffered saline (PBS), and subsequently permeated for 5 min on ice. After blocking with $1 \%$ BSA solution, samples were labeled for actin by the specific probe Alexa Fluor 488 Phalloidin (\#A12379, Thermo Scientific, Wilmington, DE, USA). Phalloidinpositive cells were counted. Results are representative of at least three independent experiments.
Quantitative real time $R T-P C R$. To validate changes in gene expression, quantitative real time RT-PCR (qPCR) analysis was performed using Applied Biosystems Prism 7900HT Sequence Detection System according to the manufacturer's instructions (Thermo Scientific, Wilmington, DE, USA) as previously described (15). Total RNA was extracted from cells using ISOGEN (Nippon Gene, Tokyo, Japan). We used SYBR Green-based detection. Reverse-transcriptase reaction was performed with High Capacity cDNA Reverse Transcription kit (Thermo Scientific, Wilmington, DE, USA). The cDNA was amplified by PCR using murine specific primers for alkaline phosphatase (ALP), bone gammacarboxyglutamic acid-containing protein (Bglap), Ntn4, and $\beta$-actin (Actb) as follows: ALP sense, 5'-GCTTTAAACCCAGACACAAG -3' and ALP antisense, 5'-GCAGTAACCACAGTCAAGGT-3'; Bglap sense, 5'-TGCTTGTGACGAGCTATCAG-3' and Bglap antisense, 5'-GAGGACAGGGAGGATCAAGT-3'; Ntn4 sense, 5'GGCCT GGAAGATGATGTTGT-3' and Ntn4 antisense, 5'-AATGG TGAGGTTTTGCGTTC-3'; Actb sense, 5'-AGAAGGACTCC TATGTGGGTGA-3' and Actb antisense, 5'-CATGATCTGGGTCAT CTTTTCA-3'. SYBR green-based qPCR was performed using THUNDERBIRD SYBR qPCR Mix (TOYOBO, Osaka, Japan). Thermal cycling conditions were conducted as previously described (15). Values were normalized to Actb using the $2^{-\Delta \Delta \mathrm{Ct}}$ method. All samples were examined in triplicate assays.

Plasmids. Mouse Ntn4 (accession number NM_021320.3) was obtained by a standard RT-PCR technique using PrimeSTAR HS DNA polymerase (TaKaRa, Ohtsu, Japan) and cloned into pcDNA3.1/V5-His expression vector (Thermo Scientific, Wilmington, DE, USA) as previously described (17).

Small interfering RNA (siRNA) transfection. RNA interference was performed as previously described (15). Stealth Select RNAi siRNA for murine Ntn4 (MSS226595: TAAAGTCCACCAAACTGA AGATCTT, MSS226596: CCACCTTTCACTCGATGCACAATAA, MSS226597: GCTCCTAACGAATGCAGAACTTGCA) and Stealth RNAi Negative Control Duplex (Low GC, Medium GC) as sicontrol (si-CTL) were purchased from Thermo Scientific (Wilmington, DE, USA). Briefly, they were all transfected with Lipofectamine RNAi MAX (Thermo Scientific, Wilmington, DE, USA) according to the manufacturer's instructions. Results were representative of more than three individual experiments.

Statistical analysis. Comparisons between two groups were analyzed using Student's t-tests and comparisons among three groups were analyzed using One-Way Analysis of Variance and Bonferroni/Dunn methods $\left({ }^{\#} p<0.05\right.$; $\left.{ }^{\# \#} p<0.01\right)$. All values are represented as the mean \pm S.E.M. Results are representative examples of more than three independent sets of experiments.

\section{Results}

Ntn4 is up-regulated during osteoblast differentiation and knockdown of endogenous Ntn4 inhibits ALP expression. We hypothesized that Ntn4 plays a vital role for osteoblast differentiation in vitro. To examine this hypothesis, we conducted qPCR to investigate the change of Ntn4 expression during osteoblast differentiation induced by ascorbic acid and $\beta$-glycerophosphate. We found that Ntn4 

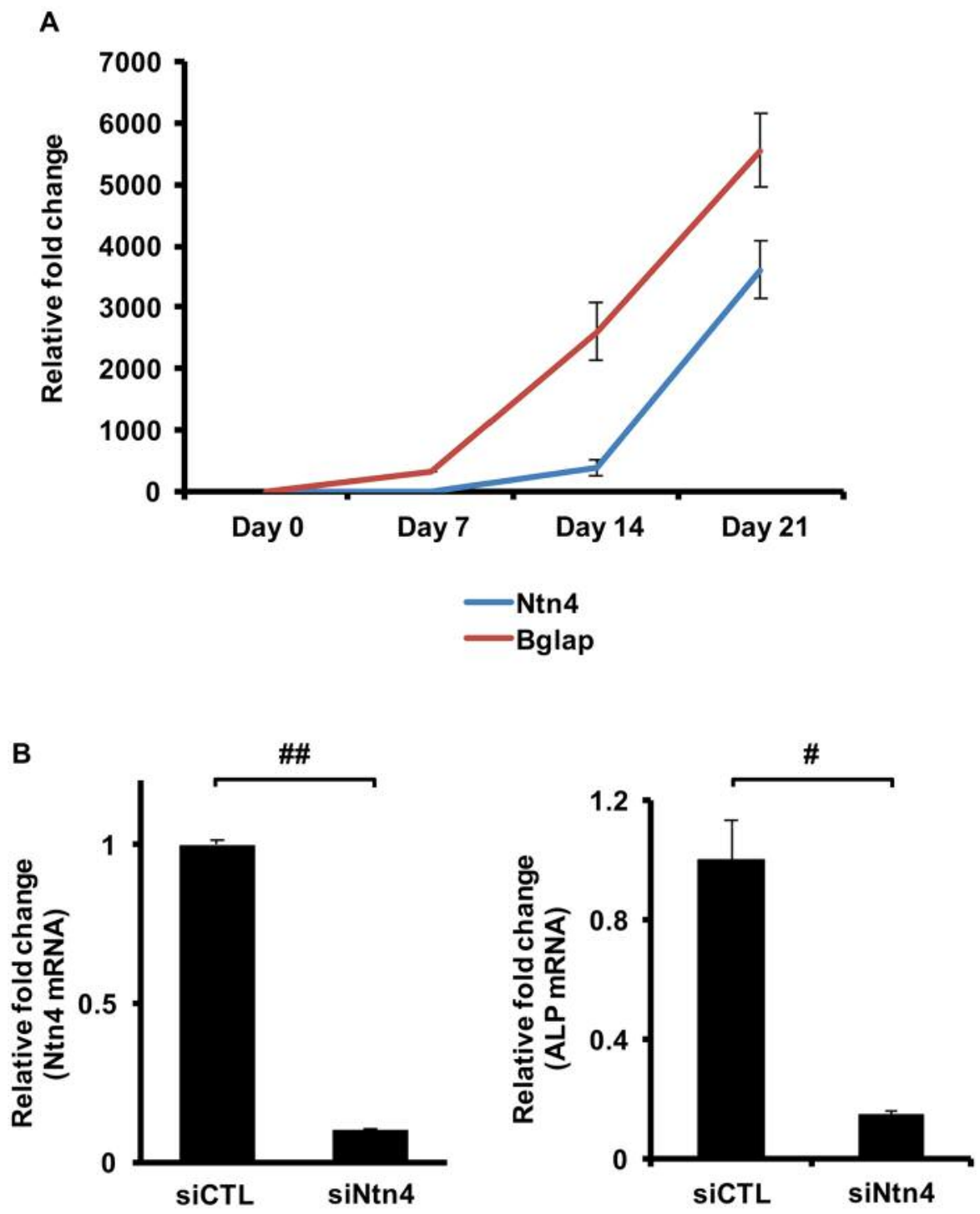

Figure 1. Ntn4 expression is essential for osteoblast differentiation. (A) The expression of Bglap and Ntn4 mRNA in MC3T3-E1 during osteoblast differentiation in the presence of ascorbic acid and $\beta$-glycerophosphate at 7 and 14, and 21 days estimated by qPCR analysis. (B) Effect of RNA interference to Ntn4 (MSS226597) in MC3T3-E1 at 3 days estimated by qPCR analysis: (left panel) Ntn4, (right panel) ALP. Data are calculated from three repeated experiments. si-control (si-CTL), Stealth RNAi Negative Control Duplex; si-Ntn4, Stealth RNAi to Ntn4. ${ }^{\# p} p 0.05$; ${ }^{\#} p<0.01$. Data are expressed as the means \pm S.E.M.

expression was up-regulated during osteoblast differentiation (Figure 1A). We next performed RNA interference of Ntn4 in MC3T3-E1. As seen in Figure 1B, we confirmed that the efficacy of gene knock-down by si-Ntn4 in MC3T3-E1 was almost $80 \sim 90 \%$ by qPCR analysis. Interestingly, RNA interference-mediated knock-down of Ntn4 in MC3T3-E1 significantly inhibited mRNA expression of ALP (Figure 1C). These results indicate that $\mathrm{Ntn} 4$ is essential for osteoblast differentiation.
Ntn4 treatment promotes both ALP activity and calcification and Ntn4 overexpression enhances ALP expression. To assess whether treatment of $\mathrm{Ntn} 4$ affects osteoblast differentiation, we added recombinant mouse Ntn4 to the culture of conditioned medium. Ntn4 promoted ALP activity of MC3T3-E1 (Figure 2A). Enhancement of calcium deposits was observed by adding Ntn4 to the culture (Figure 2B). To confirm that the increase of calcification is not due to the promotion of cell proliferation, we assessed whether Ntn4 

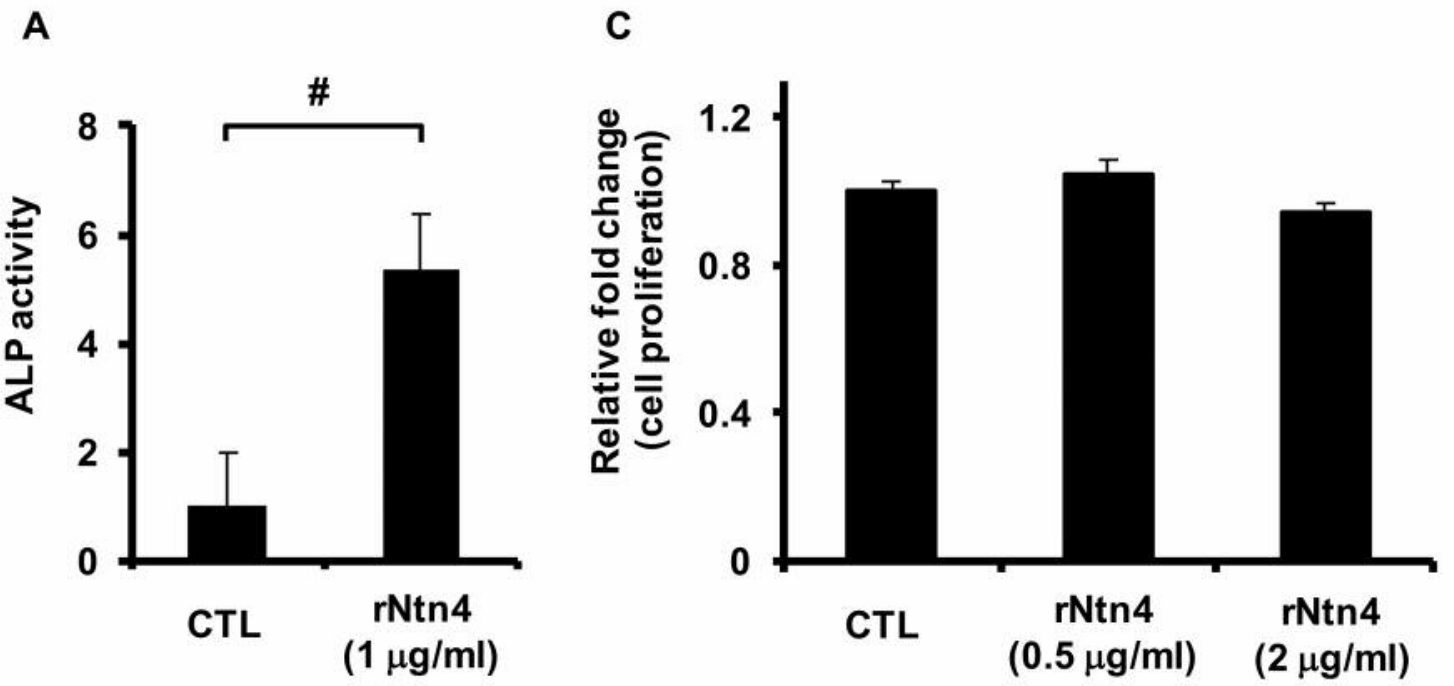

B

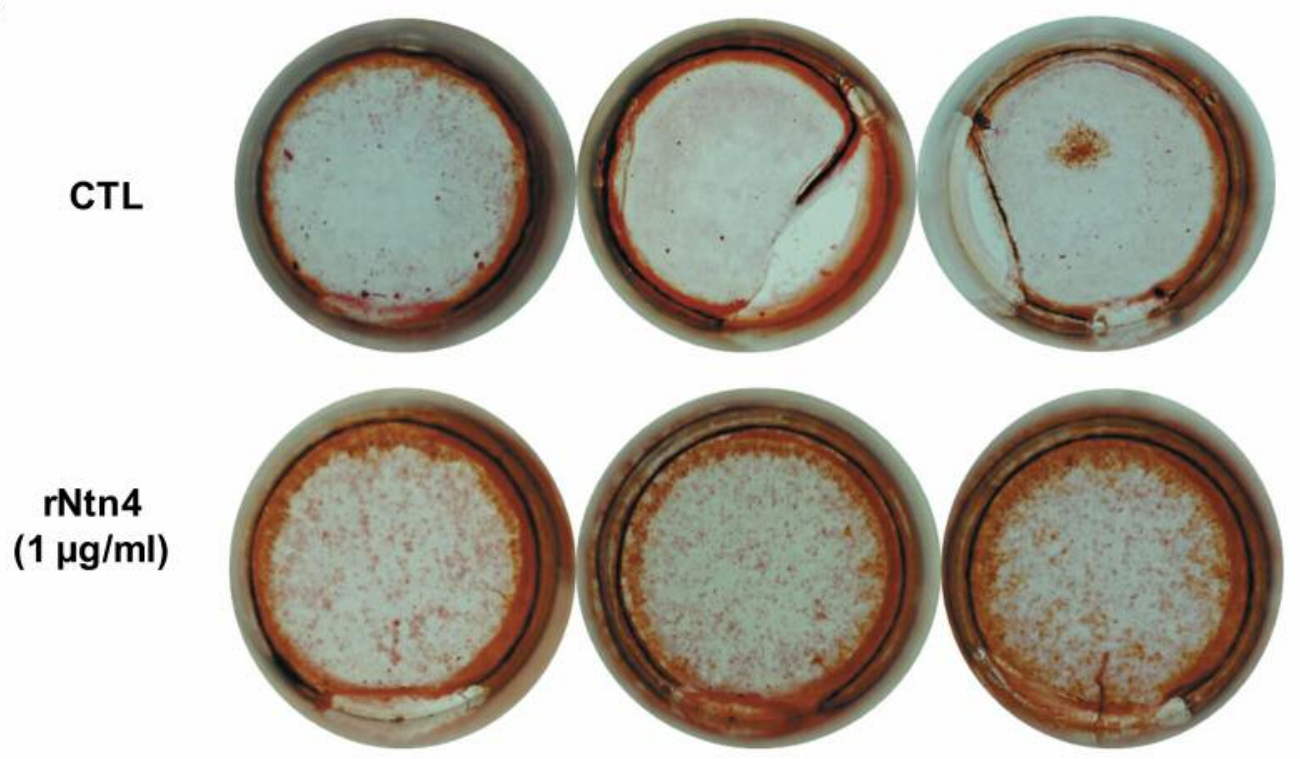

Figure 2. Recombinant Ntn4 promotes osteoblast differentiation. (A) Effect of $r N \operatorname{tn} 4(1 \mu \mathrm{g} / \mathrm{ml})$ on ALP activity of osteoblastic cells at 7 days estimated by ALP activity assay. (B) Effect of $r N \operatorname{tn} 4(1 \mu \mathrm{g} / \mathrm{ml})$ on calcium deposits at 21 days estimated by Alizarin red staining. (C) Effect of rNtn4 (0.5 and $2 \mu \mathrm{g} / \mathrm{ml}$ ) on cell proliferation of osteoblastic cells at 3 days estimated by cell proliferation assay. Data are calculated from three repeated experiments. Control, CTL. ${ }^{\#} p<0.05$. Data are expressed as the means \pm S.E.M.

affected cell proliferation in MC3T3-E1 cells. Ntn4 did not affect cell proliferation of MC3T3-E1 cells (Figure 2C).

We next examined whether Ntn4 overexpression affects osteoblast differentiation, we constructed a vector containing the Ntn4 gene on the basis of the plasmid pcDNA3.1/V5-His. As seen in Figure $3 \mathrm{~A}$, we confirmed that Ntn4 was significantly overexpressed in MC3T3-E1 cells by qPCR analysis. Overexpression of Ntn4 promoted ALP mRNA expression in the conditioned medium (Figure 3B). These results suggest that $\mathrm{Ntn} 4$ promotes osteoblast differentiation.

Ntn4 treatment promotes migration of osteoblasts. Finally, we investigated whether treatment of Ntn4 affects osteoblast migration because Ntn4 enhances migration in human 

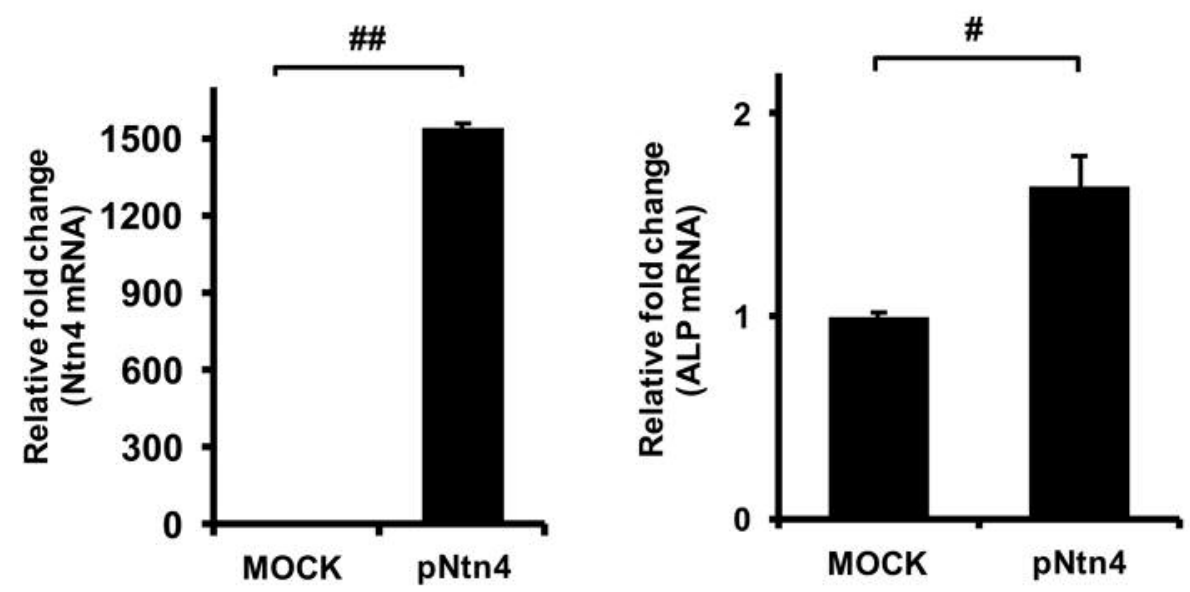

Figure 3. Ntn4 overexpression enhances osteoblast differentiation. MC3T3-E1 cells were transfected with plasmid Ntn4 (pNtn4) or an empty vector $(M O C K)$ at 3 days estimated by $q$ PCR analysis: (left panel) Ntn4, (right panel) ALP. Data are calculated from three repeated experiments. ${ }^{\#}<0.05$; $\# p<0.01$. Data are expressed as the means \pm S.E.M.

lymphatic endothelial cells (13). To establish if Ntn4 has an ability to promote cell migration, we conducted in vitro migration assay. Ntn4 treatment dramatically increased the number of MC3T3-E1 cells in the area after $12 \mathrm{~h}$ compared to the control (Figure 4) but did not affect proliferation (Figure 2C), suggesting that Ntn4 promotes migration of osteoblasts.

\section{Discussion}

The relationship between netrin receptor and bone morphogenetic protein. The present study demonstrates that Ntn4 plays a vital role not only in differentiation but also in migration of osteoblasts. Osteoblast differentiation is partly controlled by bone morphogenetic protein (BMP) (18). Recent studies have shown that BMP is the key molecule connecting netrin with osteoblastic differentiation. Neogenin, which belongs to one of the netrin receptors, is involved in the regulation of BMP-induced Smad signaling and endochondral bone formation (19). Their studies provide evidence that neogenin may regulate chondrocyte maturation by promoting BMP induced BMP receptor association with lipid rafts, thus enhances effective BMP receptor concentration or BMP binding affinity and increases Smad phosphorylation and Runx 2 induction by using neogenin deficient mice, suggesting that neogenin promotes chondrogenesis in vitro and in vivo. However, a controversial result has been reported. Hagihara et al. reported that neogenin negatively regulates the functions of BMP and that this effect of neogenin is mediated by the activation of RhoA (20). However, it is conjectured that the inhibition of osteoblast differentiation by BMP via the activation of neogenin may not occur under physiological conditions.

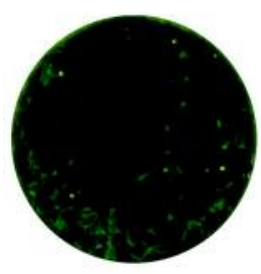

CTL

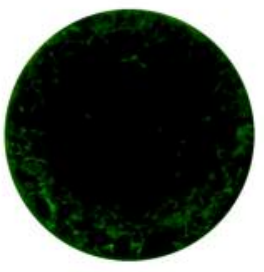

rNtn4

$(0.5 \mu \mathrm{g} / \mathrm{ml})$

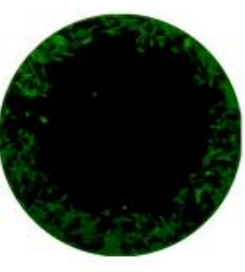

rNtn4 $(2 \mu \mathrm{g} / \mathrm{ml})$

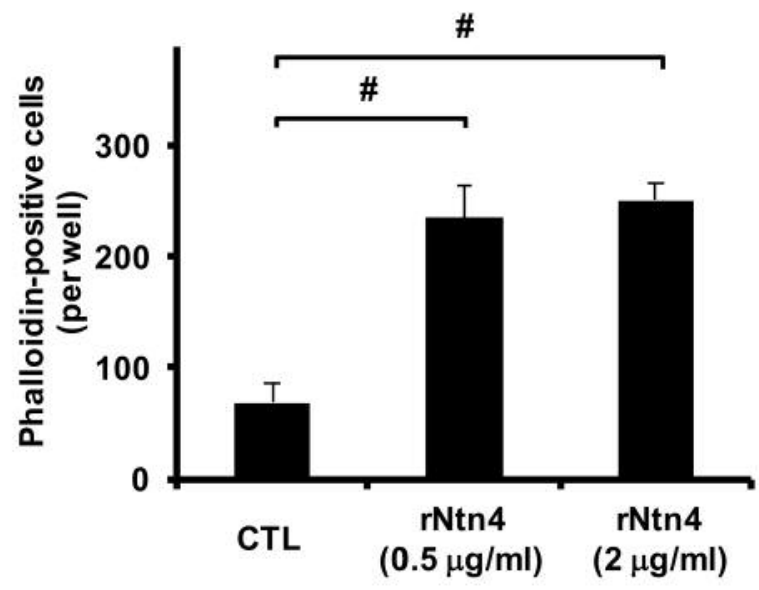

Figure 4. Recombinant Ntn4 enhances osteoblast migration. Effect of rNtn4 $(0.5$ and $2 \mu \mathrm{g} / \mathrm{ml})$ on cell migration of osteoblastic cells at $12 \mathrm{~h}$ estimated by cell migration assay: (upper panel) Ntn4, (lower panel) ALP. Data are calculated from three repeated experiments. Control, $C T L .{ }^{\#} p<0.05$. Data are expressed as the means \pm S.E.M. 
The differentiation in various cells is associated with Ntn4. $\mathrm{Ntn} 4$ also has a functional role in the differentiation process of other cells. Vascular and ductal cells in the developing human pancreas produce Ntn4 to maintain islet differentiation (21). We have previously demonstrated that $\mathrm{Ntn} 4$, that is derived from vascular endothelial cells, inhibits osteoclast differentiation (15). Osteoblasts are essentially involved in the differentiation of osteoclasts through a mechanism involving cell-to-cell contact between osteoblasts and osteoclast precursors (22). We speculate that Ntn4 derived from osteoblasts may play a role as a brake of osteoclast differentiation through cell-to-cell contact.

The migration in various cells is associated with Ntn4. Ntn4 is involved in the migration of various cells. Ntn4 promotes mural cell adhesion and recruitment to endothelial cells (23). In Müller cells, the MAP kinase pathway is essential for the migration by Ntn4 which contributes to angiogenesis in the retina (24). Ntn4-stimulated phosphorylation of the Src kinase family, effectors of endothelial cell migration, is also abolished by $\alpha 6$ or $\beta 1$ inhibition (25). Ntn4 induces proliferation, migration and survival of lymphatic endothelial cells through activation of p42/p44 MAP kinase, Akt/PI3kinase and mTor signaling pathways (13). In osteoblasts, TGF- $\beta$ stimulates vascular endothelial growth factor synthesis which promotes the migration (26) and Nck (noncatalytic region of tyrosine kinase), that is a member of the signaling adaptors that control remodeling of the actin cytoskeleton, regulates cell migration (27). In this study, for the first time, we showed that Ntn4 promotes cell migration of osteoblasts. We speculate that Ntn4 may play a role at the site of bone remodeling or modeling in order to maintain homeostasis.

The relationship between other netrins and bone metabolism. Togari et al. showed that netrin-1 (Ntn1), netrin-2-like protein, and netrin-3 mRNA were expressed by osteoblasts and also found that netrin-3 mRNA was expressed by osteoclasts (28). Yagami et al. found that the expression of Ntn 1 was decreased in the process whereby mesenchymal cells differentiate into osteoblasts by BMP (29).

It has been reported that Ntn1 is involved in regulating bone remodeling. Mediero et al. demonstrated that Ntn 1 , which is produced by osteoclasts, enhanced osteoclast differentiation by autocrine/paracrine manner and Ntn1 deficient mice have markedly diminished osteoclasts, as well as increased cortical and trabecular bone density and volume compared with wild type mice, suggesting that $\mathrm{Ntn} 1$ is a negative regulator of bone metabolism (8). In contrast, Maruyama et al. reported that Ntn1 suppresses osteoclast multinucleation, but not osteoclast differentiation and protected mice against autoimmune bone destruction in vivo, indicating that $\mathrm{Ntn} 1$ is a positive regulator in bone metabolism (9).
Recently, we found that Ntn1 inhibits osteoblast differentiation and the combination of two netrin receptors is essential for regulating osteoblast differentiation by Ntn1 (30). Further investigations will be required to clarify the role of netrins in bone metabolism.

In conclusion, we found that Ntn4 promotes differentiation and migration of osteoblasts.

\section{Acknowledgements}

This work was supported by Grants-in-Aid for Scientific Research from the Ministry of Education, Science, Sports and Culture of Japan (15K20556 to Yuichiro Enoki).

\section{References}

1 Delaisse JM: The reversal phase of the bone-remodeling cycle: cellular prerequisites for coupling resorption and formation. Bonekey Rep 3: 561, 2014.

2 Maes C: Role and regulation of vascularization processes in endochondral bones. Calcif Tissue Int 92: 307-323, 2013.

3 Houweling P, Kulkarni RN and Baldock PA: Neuronal control of bone and muscle. Bone 80: 95-100, 2015.

4 Fukuda T, Takeda S, Xu R, Ochi H, Sunamura S, Sato T, Shibata S, Yoshida Y, Gu Z, Kimura A, Ma C, Xu C, Bando W, Fujita K, Shinomiya K, Hirai T, Asou Y, Enomoto M, Okano H, Okawa $\mathrm{A}$ and Itoh $\mathrm{H}$ : Sema3 A regulates bone-mass accrual through sensory innervations. Nature 497: 490-493, 2013.

5 Hayashi M, Nakashima T, Taniguchi M, Kodama T, Kumanogoh A and Takayanagi H: Osteoprotection by semaphorin 3A. Nature 485: 69-74, 2012.

6 Delorme G, Saltel F, Bonnelye E, Jurdic P and Machuca-Gayet I: Expression and function of semaphorin 7A in bone cells. Biol Cell 97: 589-597, 2005.

7 Negishi-Koga T, Shinohara M, Komatsu N, Bito H, Kodama T, Friedel RH and Takayanagi H: Nat Med 17: 1473-1480, 2011.

8 Mediero A, Ramkhelawon B, Perez-Aso M, Moore KJ and Cronstein BN: Netrin-1 is a critical autocrine/paracrine factor for osteoclast differentiation. J Bone Miner Res 30: 837-854, 2015.

9 Maruyama K, Kawasaki T, Hamaguchi M, Hashimoto M, Furu M, Ito H, Fujii T, Takemura N, Karuppuchamy T, Kondo T, Fukasaka M, Misawa T, Saitoh T, Suzuki Y, Martino MM, Kumagai Y and Akira S: Bone-protective Functions of Netrin 1 Protein. J Biol Chem 291: 23854-23868, 2016.

10 Rajasekharan S and Kennedy TE: The netrin protein family. Genome Biol 10: 239, 2009.

11 Eveno C, Contreres JO, Hainaud P, Nemeth J, Dupuy E and Pocard M: Netrin-4 overexpression suppresses primary and metastatic colorectal tumor progression. Oncol Rep 29: 73-78, 2013.

12 Kociok N, Crespo-Garcia S, Liang Y, Klein SV, Nürnberg C, Reichhart N, Skosyrski S, Moritz E, Maier AK, Brunken WJ, Strauß O, Koch M and Joussen AM: Lack of netrin-4 modulates pathologic neovascularization in the eye. Sci Rep 6: 18828, 2016.

13 Larrieu-Lahargue F, Welm AL, Thomas KR and Li DY: Netrin4 induces lymphangiogenesis in vivo. Blood 115: 5418-5426, 2010. 
14 Esseghir S1, Kennedy A, Seedhar P, Nerurkar A, Poulsom R, Reis-Filho JS and Isacke CM: Identification of NTN4, TRA1, and STC2 as prognostic markers in breast cancer in a screen for signal sequence encoding proteins. Clin Cancer Res 13: 31643173, 2007.

15 Enoki Y, Sato T, Tanaka S, Iwata T, Usui M, Takeda S, Kokabu S, Matsumoto M, Okubo M, Nakashima K, Yamato M, Okano T, Fukuda T, Chida D, Imai Y, Yasuda H, Nishihara T, Akita M, Oda H, Okazaki Y, Suda T and Yoda T: Netrin-4 derived from murine vascular endothelial cells inhibits osteoclast differentiation in vitro and prevents bone loss in vivo. FEBS Lett 588: 2262-2269, 2014.

16 Sato T, Abe T, Nakamoto N, Tomaru Y, Koshikiya N, Nojima J, Kokabu S, Sakata Y, Kobayashi A and Yoda T: Nicotine induces cell proliferation in association with cyclin D1 up-regulation and inhibits cell differentiation in association with p53 regulation in a murine pre-osteoblastic cell line. Biochem Biophys Res Commun 377: 126-130, 2008.

17 Kokabu S, Nguyen T, Ohte S, Sato T, Katagiri T, Yoda T and Rosen V: TLE3, transducing-like enhancer of split 3, suppresses osteoblast differentiation of bone marrow stromal cells. Biochem Biophys Res Commun 438: 205-210, 2013.

$18 \mathrm{Wu}$ M, Chen $\mathrm{G}$ and Li YP: TGF- $\beta$ and BMP signaling in osteoblast, skeletal development, and bone formation, homeostasis and disease. Bone Res 4: 16009, 2016.

19 Zhou Z, Xie J, Lee D, Liu Y, Jung J, Zhou L, Xiong S, Mei L and Xiong WC: Neogenin regulation of BMP-induced canonical Smad signaling and endochondral bone formation. Dev Cell 19: 90-102, 2010.

20 Hagihara M, Endo M, Hata K, Higuchi C, Takaoka K, Yoshikawa $\mathrm{H}$ and Yamashita T: Neogenin, a receptor for bone morphogenetic proteins. J Biol Chem 286: 5157-5165, 2011.

21 Yebra M, Diaferia GR, Montgomery AM, Kaido T, Brunken WJ, Koch M, Hardiman G, Crisa L and Cirulli V: Endotheliumderived Netrin-4 supports pancreatic epithelial cell adhesion and differentiation through integrins $\alpha 2 \beta 1$ and $\alpha 3 \beta 1$. PLoS One 6 : e22750, 2011.

22 Udagawa N, Takahashi N, Jimi E, Matsuzaki K, Tsurukai T, Itoh K, Nakagawa N, Yasuda H, Goto M, Tsuda E, Higashio K, Gillespie MT, Martin TJ and Suda T: Osteoblasts/stromal cells stimulate osteoclast activation through expression of osteoclast differentiation factor/RANKL but not macrophage colonystimulating factor: receptor activator of NF-kappa B ligand. Bone 25: 517-523, 1999.
23 Lejmi E, Bouras I, Camelo S, Roumieux M, Minet N, Leré-Déan C, Merkulova-Rainon T, Autret G, Vayssettes C, Clement O, Plouët $\mathrm{J}$ and Leconte L: Netrin-4 promotes mural cell adhesion and recruitment to endothelial cells. Vasc Cell 6: 1, 2014.

24 Lange J, Yafai Y, Noack A, Yang XM, Munk AB, Krohn S, Iandiev I, Wiedemann P, Reichenbach A and Eichler W: The axon guidance molecule Netrin-4 is expressed by Müller cells and contributes to angiogenesis in the retina. Glia 60: 1567$1578,2012$.

25 Larrieu-Lahargue F, Welm AL, Thomas KR and Li DY: Netrin4 activates endothelial integrin \{alpha\}6\{beta\}1. Circ Res 109: 770-774, 2011.

26 Yamamoto N, Tokuda H, Kuroyanagi G, Kainuma S, Matsushima-Nishiwaki R, Fujita K, Kozawa O and Otsuka T: Heat shock protein 22 (HSPB8) limits TGF- $\beta$-stimulated migration of osteoblasts. Mol Cell Endocrinol 436: 1-9, 2016.

27 Aryal ACS, Miyai K, Izu Y, Hayata T, Notomi T, Noda M and Ezura Y: Nck influences preosteoblastic/osteoblastic migration and bone mass. Proc Natl Acad Sci USA 112: 15432-15437, 2105.

28 Togari A, Mogi M, Arai M, Yamamoto S and Koshihara Y: Expression of mRNA for axon guidance molecules, such as semaphorin-III, netrins and neurotrophins, in human osteoblasts and osteoclasts. Brain Res 878: 204-209, 2000.

29 Yagami K, Nakamura M, Nakamura H, Maki S, Yagasaki T and Udagawa N: Neural-inducing Factor Netrin-1 is Regulated in Chondrogenesis and Osteogenesis by BMP or Noggin. J Hard Tissue Biol 21: 141-150, 2012.

30 Sato T, Kokabu S, Enoki Y, Hayashi N, Matsumoto M, Nakahira M, Sugasawa M and Yoda T: Functional Roles of Netrin-1 in Osteoblast Differentiation. In Vivo 31: 321-328, 2017.
Received May 16, 2017

Revised May 27, 2017

Accepted May 29, 2017 